\section{Alteraciones electrocardiográficas en hipotermia severa accidental. Caso clínico y revisión de la literatura}

\author{
PILAR MUÑOZ B. ${ }^{a}$, VÍCTOR ROSSEL M.
}

\section{Electrocardiographic manifestations of severe accidental hypothermia. Report of one case}

We report a 77-year-old female patient who was admitted to the Emergency Department with impairment of consciousness, hypotension, bradycardia and hypothermia. She required endotracheal intubation and transfer to Intensive Care Unit (ICU). Computed tomography of the brain showed no lesions. Electrocardiogram showed abnormalities suggestive of severe hypothermia (bradycardia, marked elevation of J point associated with ST depression, a negative T wave in V2 to V6 and prolongation of QTc), which was confirmed with a pulmonary artery catheter. Myxedema coma, infections and neurological diseases were discarded. The cause of severe hypothermia was unclear, and the probable source was suspected to be accidental. After intensive treatment the patient improved, achieving normalization of electrocardiographic changes, recovery of organic functions and she was discharged home after 22 days.

(Rev Med Chile 2014; 142: 1205-1209)

Key words: Aged; Electrocardiography; Hypothermia.

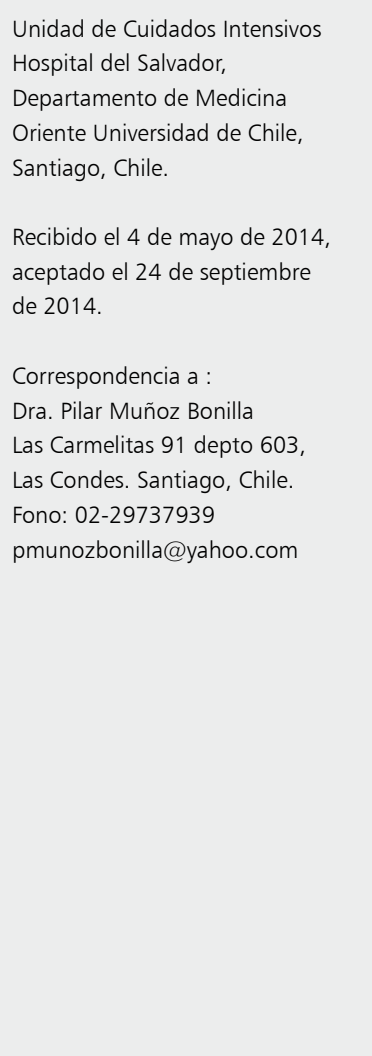

S e define hipotermia como temperatura corporal central menor a $35^{\circ} \mathrm{C}$, según su severidad, se clasifica en leve $\left(\geq 32^{\circ} \mathrm{C} y<35^{\circ} \mathrm{C}\right)$, moderada $\left(\geq 28^{\circ} \mathrm{C}\right.$ y $\left.<32^{\circ} \mathrm{C}\right)$ y severa $\left(<28^{\circ} \mathrm{C}\right)^{1}$.

Existen múltiples etiologías de hipotermia y en lo fisiopatológico se describen 4 mecanismos productores: 1) aumento de pérdidas de calor por evaporación, radiación, conducción y convección (por exposición ambiental accidental y uso de fármacos o toxinas que producen vasodilatación);2) disminución de producción de calor (hipotiroidismo, insuficiencia suprarrenal, hipoglicemia); 3) alteraciones de termorregulación (enfermedades que afectan el sistema nervioso central); y 4) otros (sepsis y trauma). Este cuadro produce anormalidades a nivel celular, destacando alteraciones de propiedades de membranas y sus proteínas, salida de componentes del intracelular al extracelular, desbalances hidroelectrolíticos y disfunción enzimática, los cuales explican las complicaciones sistémicas observadas ${ }^{2}$.

La hipotermia accidental en adultos es un cua- dro de baja incidencia y el principal mecanismo productor es la pérdida de calor por conducción y convección. El manejo de las complicaciones es complejo y la mortalidad intrahospitalaria, de los casos moderados y severos, se estima en $40 \%$ a pesar de cuidados intensivos modernos ${ }^{3}$. Los adultos mayores tienen mayor riesgo de desarrollar este evento, por disminución de reserva fisiológica, comorbilidades crónicas, uso de medicamentos que impiden respuestas compensatorias y aislamiento social ${ }^{4}$.

Desde el punto de vista cardiológico, las manifestaciones de hipotermia moderada y severa incluyen bradicardia, hipotensión arterial, bajo gasto cardiaco, fibrilación auricular (FA), arritmias ventriculares (fibrilación ventricular $[\mathrm{FV}]$ ) y asistolía ${ }^{5}$. En el electrocardiograma (EKG) se describen alteraciones características que permiten sospechar la hipotermia.

El objetivo de presentar este caso clínico es revisar las alteraciones electrocardiográficas que produce la hipotermia, para así no errar el diagnóstico ni retrasar el tratamiento. 


\section{Caso clínico}

Paciente de género femenino de 77 años, con antecedentes de cardiopatía coronaria. Encontrada inconsciente en domicilio por familiares. Evaluada en Servicio de Urgencias del Hospital del Salvador, se constató presión arterial: 97/46 $\mathrm{mmHg}$, presión arterial media: $57 \mathrm{mmHg}$, frecuencia cardiaca: $47 \mathrm{lpm}$, oximetría de pulso: $85 \%$ con oxígeno ambiental, no logrando sensar temperatura con termómetro de mercurio y en Glasgow 6. Al examen físico general se describió en malas condiciones generales, enflaquecida, fría, mal perfundida y con presencia de livideces generalizadas. En lo segmentario destacaba presencia de bocio difuso, examen cardiaco con ritmo irregular por extrasistolía ventricular frecuente y examen pulmonar con estertores basales escasos. Al examen físico neurológico en sopor profundo reactiva sólo al estímulo doloroso, miosis puntiforme bilateral fotorreactiva, reflejos de tronco indemnes, sin focalidad evidente y signos meníngeos ausentes. Se procedió a intubación orotraqueal para protección de vía aérea y se inició estudio complementario. En exámenes de laboratorio se constató glicemia normal, leucopenia $\left(2.020 \mathrm{~mm}^{3}\right.$, sin alteraciones de fórmula diferencial), hipokalemia $(2,6 \mathrm{mmol} / \mathrm{L})$, creatinina: 0,69 mg/dl, nitrógeno ureico: $19 \mathrm{mg} /$ $\mathrm{dl}$, gasometría arterial en rango normal, lactato $13 \mathrm{mg} / \mathrm{dl}$, creatinquinasa: $222 \mathrm{U} / \mathrm{L}$, creatinquinasa MB: $113 \mathrm{U} / \mathrm{L}$ y troponina I: 0,02 ug/L. EKG de ingreso evidenció bradicardia sinusal de $42 \mathrm{lpm}$, eje eléctrico normal, conducción aurículoventricular conservada, elevación marcada del punto J asociada a infradesnivel del segmento ST con onda T negativa en las derivaciones precordiales $\mathrm{V} 2$ a V6 y prolongación del intervalo QTc (Figura 1). TAC de cerebro sin alteraciones que explicaran cuadro clínico. Ingresó a UCI conectándose a ventilación mecánica invasiva e inició apoyo con noradrenalina hasta $0,35 \mathrm{ug} / \mathrm{kg} / \mathrm{min}$. Se instaló catéter de arteria pulmonar, obteniéndose presión venosa central (PVC): $8 \mathrm{mmHg}$, presión de oclusión de capilar pulmonar (PAOP): $11 \mathrm{mmHg}$ y temperatura cor-

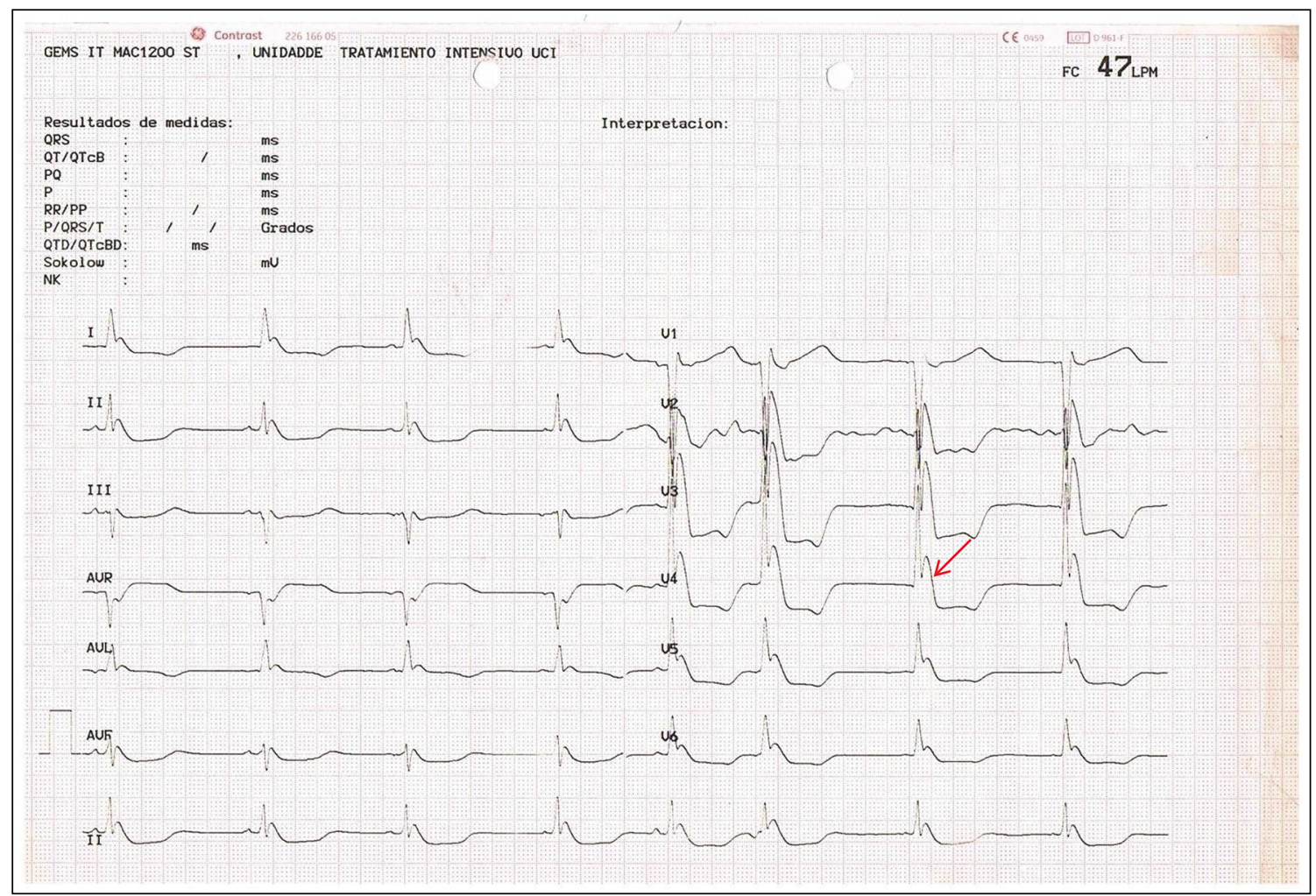

Figura 1. EKG de ingreso. Se observa bradicardia sinusal, conducción aurículo ventricular (AV) conservada, Onda J de Osborn (flecha roja), infradesnivel del segmento ST y onda T negativa mayor en derivaciones precordiales, y segmento QTc prolongado (620 mseg). 
poral central de $27,9^{\circ} \mathrm{C}$ que no permitió calcular gasto cardiaco por método de termodilución. Se diagnosticó hipotermia severa y se inició tratamiento con medidas de recalentamiento externas e internas (manta térmica y aportes de cristaloides tibios por vía enteral y parenteral) y corrección de hipokalemia. En el estudio etiológico se descartó foco infeccioso, coma mixedematoso, hipoglicemia y causa neurológica. No se dispuso de laboratorio para descartar intoxicación farmacológica ni insuficiencia suprarrenal, sin embargo, la evolución clínica descarta razonablemente estos diagnósticos. La curva de marcadores séricos de necrosis miocárdica fue negativa. Normalizada la temperatura corporal $(48 \mathrm{~h}$ posterior a ingreso a UCI) la paciente evolucionó favorablemente, con recuperación total de conciencia sin secuelas neurológicas y con regresión completa de las alteraciones del EKG (Figura 2). Ecoscopia cardiaca (realizada al quinto día de hospitalización) informó función ventricular izquierda estimada en $55 \%$, akinesia adelgazada de pared inferior en segmento medio lateral del ventrículo izquierdo, ventrículo derecho sin alteraciones de motilidad y ausencia de reflujos valvulares. A los 12 días de su estadía hospitalaria, presentó episodio de FA de respuesta ventricular rápida sin compromiso hemodinámico, que convirtió con amiodarona (bolo de $300 \mathrm{mg}$ ). Logró alta a domicilio en buenas condiciones tras 22 días de hospitalización.

\section{Discusión}

La hipotermia produce alteraciones sistémicas graves. A nivel cardiaco, deprime la actividad de la bomba de calcio dependiente de ATP (Ca- ATPasa) disminuyendo el número de unidades activas en la membrana celular. Esto produce retardo en el influjo de calcio que prolonga la duración del potencial de acción, disminuye el automatismo de las células marcapasos y enlentece la conducción del impulso miocárdico. Esto provoca anormalidades de repolarización que se traducen

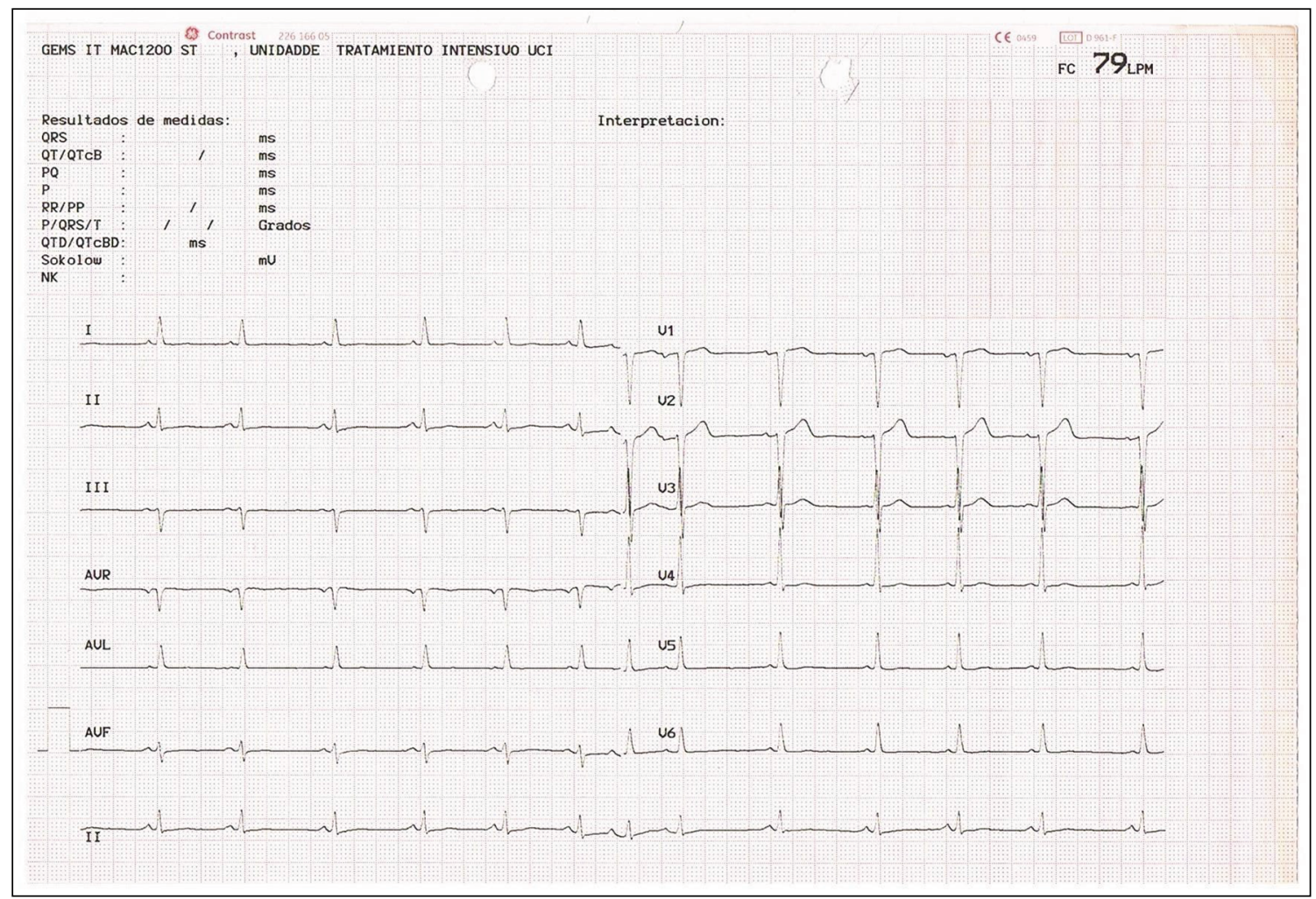

Figura 2. EKG post corrección de hipotermia. Se observa regresión completa de las alteraciones evidenciadas en la figura 1 al normalizar la temperatura corporal, no evidenciándose secuelas electrocardiográficas. 
en alteraciones electrocardiográficas clásicas que incluyen: prolongación de intervalos PR-QRSQT, elevación del punto J y aparición de arritmias tanto auriculares como ventriculares. Además, la disminución del calcio a nivel intracelular en los miocardiocitos altera la contractilidad y reduce el gasto cardiaco ${ }^{6,7}$.

El caso presentado evidencia en el EKG las alteraciones más frecuentes observadas en hipotermia severa. De éstas, la más estudiada es la onda J, descrita por primera vez en 1938 por Tomasjewski, sin embargo, fue Osborn quien investigó los posibles mecanismos de esta alteración en 1953 y debido a él recibe su nombre actual (onda de Osborn). Corresponde a la deflección positiva de la porción terminal del complejo QRS con elevación del punto J, que se encuentra frecuentemente en derivaciones precordiales anteriores y laterales, pudiendo ser confundida con bloqueo completo de rama derecha. La causa exacta de esta alteración es desconocida, pero se postula la existencia de una diferencia de voltaje entre endocardio y epicardio en la repolarización ventricular temprana, producida por una mayor salida de potasio hacia el extracelular, lo que altera la morfología del potencial de acción ${ }^{8,9}$. Usualmente se presenta hasta en $80 \%$ de los casos de hipotermia moderada/ severa, siendo de alta sensibilidad y especificidad para sospecha y diagnóstico. Su tamaño es inversamente proporcional a la temperatura corporal, sin embargo, no se ha podido demostrar correlación de ésta con las alteraciones electrolíticas y ácido base. A pesar de la frecuencia con la que se presenta la onda de Osborn en estos casos, ésta carece de valor pronóstico e incluso no es patognomónica de hipotermia, habiéndose descrito también en hemorragia subaracnoídea y otras lesiones cerebrales, isquemia miocárdica, hipercalcemia y en pacientes jóvenes normotérmicos con repolarización precoz ${ }^{10}$.

La hipotermia se asocia además a variadas arritmias auriculares y ventriculares, siendo la más frecuente la FA. En hipotermia moderada/ severa, se presenta en hasta $50 \%$ de los casos, sin embargo, su aparición no se correlaciona con peor pronóstico. Frecuentemente convierte en forma espontánea durante la fase de recalentamiento o una vez normalizada la temperatura corporal y en general, no necesita tratamiento antiarrítmico ni anticoagulante (hipotermia severa produce coagulopatía severa per se). Los casos más seve- ros tienen riesgo elevado de FV y asistolía que son refractarias al manejo con desfibrilaciones y fármacos mientras se mantenga la hipotermia ${ }^{10}$. Es importante destacar que todas las alteraciones electrocardiográficas y las arritmias revierten al corregir la hipotermia.

El caso expuesto muestra las alteraciones electrocardiográficas descritas, la relación entre severidad de la hipotermia y magnitud de la onda de Osborn y la reversibilidad de las anormalidades electrocardiográficas. Cabe destacar que existía concomitantemente hipokalemia significativa, la cual por sí sola se asocia a alteraciones electrocardiográficas específicas (prolongación del segmento QT, infradesnivel del segmento ST y bradicardia sinusal, entre otras), todas presentes en los EKG de la paciente y que en este caso, podrían ser sumatorias. De todas formas llama la atención que a pesar de la hipotermia severa y la hipokalemia, en el EKG no se observaron alteraciones del intervalo PR. En su estadía en UCI presentó extrasístoles ventriculares frecuentes antes de iniciar el recalentamiento, pero no arritmias ventriculares malignas. Afortunadamente, a pesar de las estadísticas de sobrevida desfavorables, la paciente respondió al tratamiento y se recuperó sin secuelas neurológicas ni cardiacas. Sin embargo, no se logró identificar con claridad la causa de la hipotermia, sospechándose como probable causa una exposición ambiental accidental en un paciente con factores de riesgo para desarrollar el cuadro.

Consideramos que es importante que los médicos conozcan y estén familiarizados con este tipo de alteraciones electrocardiográficas. No es infrecuente que la onda de Osborn se confunda con otros diagnósticos, y su mala interpretación retrasará el diagnóstico y tratamiento, afectando directamente el pronóstico. No hay que olvidar que la hipotermia corresponde a una manifestación de una causa subyacente, por lo que se deben agotar los esfuerzos para identificar la etiología de la hipotermia para corregir la causa desencadenante.

\section{Referencias}

1. Jolly BT, Ghezzi KT. Accidental hypothermia. Emerg Med Clin North Am 1992; 10 (2): 311.

2. Hanania Na, Zimmerman JL. Accidental hypothermia. Crit Care Clin 1999; 15 (2): 235-49. 
3. Vassal T, Benoit-Gonin B, Carrat F, Guidet B, Maury E, Offenstadt G. Severe accidental hypothermia treated in an ICU: prognosis and outcome. Chest 2001; 120 (6): 1998.

4. Ballester JM, Harchelroad FP. Hypothermia: an easy-tomiss, dangerous disorder in winter weather. Geriatrics 1999; 54 (2): 51.

5. Danzi DF, Pozos RF. Accidental hypothermia. N Engl J Med 1994; 331 (26): 1756-1760.

6. Wang H, Hollingsworth J, Mahler S. Diffuse ST segment depression fron hypothermia. Int J Emerg Med 2012; 3:
451-4.

7. Ahmed F, Ahmad K, Balendu C, Ijaz A. Hypothermia: Evaluation, Electrocardiographic manifestations, and Management. Am J Med 2006; 119 (4): 297-301.

8. Krantz M, Lowery C. Giant Osborn waves in hypothermia. N Engl J Med 2005; 352 (2): 184.

9. Yan G, Antzelevitch C. Cellular basis for the electrocardiographic J wave. Circulation 1996; 93: 372-9.

10. Mattu A, Brady W, Perron A. Electrocardiographic manifestations of Hypothermia. Am J Emerg Med 2002; 20 (4): 314-26. 